J. Dairy Sci. 92:2166-2173
doi:10.3168/jds.2008-1324
(c) American Dairy Science Association, 2009.

\title{
Graphical approach to evaluate genetic estimates of calf survival ${ }^{1}$
}

\author{
H. N. Schlesser, ${ }^{*}$ R. D. Shanks, ${ }^{* 2}$ P. J. Berger, $†$ and M. H. Healey† \\ *Department of Animal Sciences, University of Illinois, Urbana 61801 \\ †Department of Animal Science, lowa State University, Ames 50011
}

\section{ABSTRACT}

Genetic variation and resemblance among relatives are fundamentals of quantitative genetics. Our purpose was to identify bulls with a bimodal pattern of inheritance in the quest for new discoveries about the inheritance of calf survival. A bimodal pattern of inheritance for calf survival was identified in sons of Holstein bulls. A bimodal pattern of inheritance indicates 2 groups of sons resulting from an allele effect, a grandsire effect, or some other common factor. Different combinations (AA, Aa, aa) of 2 alleles at a locus cause varying phenotypes to be expressed. Bulls that are heterozygous for loci affecting reproductive performance may have a bimodal pattern of inheritance if the difference in effect of the 2 alleles is large. If the bimodal pattern is caused by an allele effect, then molecular markers can be identified for use in marker-assisted selection breeding programs. Data on predicted transmitting ability for perinatal survival for the first parity of 8,678 sons of 599 sires were collected from 1984 through 1997 from the National Association of Animal Breeders calving ease database, which included 7 Midwestern states. Sixteen bulls were identified with a potential bimodal pattern of inheritance because they had 2 distinct groups of sons. The 2 groups of sons were separated by calculating the coefficient of variation for each possible combination of sons; the combination that gave the smallest coefficient of variation difference between the 2 groups was considered the correct distribution of the sons into those groups. Bulls with a bimodal distribution were analyzed to determine the distribution of the grandsons among the maternal grandsires (MGS) of the 2 groups of the bimodal distribution. The bimodal distribution may be a result of heterozygous sires or MGS that are homozygous for low or high survival. If the bimodal distribution is caused by a MGS effect, then

Received May 2, 2008

Accepted December 10, 2008

${ }^{1}$ This research was partially supported by USDA-Cooperative State Research, Education, and Extension Service Formula Funds through Multi-State Project S-1008 and S-1040.

${ }^{2}$ Corresponding author: rdshanks@illinois.edu marker-assisted selection can still be used by evaluating the MGS instead of the sires.

Key words: calf survival, coefficient of variation

\section{INTRODUCTION}

Perinatal survival affects many dairy producers in the United States. Recently, stillbirth has described as calf loss within $48 \mathrm{~h}$ of birth (Cole et al., 2007a,b). In this paper, the term perinatal survival is used to emphasize that the bimodal results were found for PTA calculated by Meyer et al. (2001b) and not the USDA analyses (Cole et al., 2007a,b). Predicted transmitting ability of perinatal survival was based on first-parity estimates only. The statistical description of the data can be found in Meyer et al. (2001b) and was summarized and compared with more recent results by Cole et al. (2007a). Therefore, results of this study on perinatal survival may be different from results of the same methods applied to stillbirths.

Meyer et al. (2000) estimated that annually about $7 \%$ of the Holstein calves born in the United States die within $48 \mathrm{~h}$ of birth. Thompson et al. (1981) estimated that the loss from stillbirths in the United States was $\$ 92$ million dollars from 11 million calvings. These expenses included loss of calf or cow, veterinary fees, increased labor costs, and future fertility problems. Estimates of calf loss showed that $57 \%$ of all losses up to weaning occurred during the first $24 \mathrm{~h}$ postpartum and $66 \%$ occurred by $48 \mathrm{~h}$ postpartum (Patterson et al., 1987). Perinatal survival was defined to include all live calves at $48 \mathrm{~h}$ postpartum (Meyer et al., 2001a). Meyer et al. (2001a) reported that the incidence of stillbirths has steadily increased from 1985 to 1996 . The rate of increase differs between primiparous cows and multiparous cows. Over an 11-yr study period from 1985 to 1996, the percentage of stillborn calves in primiparous cows increased from 9.5 to $13.2 \%$, or $3.7 \%$. Stillbirth in multiparous cows increased from 5.3 to $6.6 \%$ or $1.3 \%$ (Meyer et al., 2001a). A logistic regression model was fit with fixed and random effects to estimate the amount of stillbirth in an average herd. From this model, it was found that the expected probability of stillbirths was $10 \%$ for primiparous cows and $5 \%$ for multiparous 
Table 1. Distribution of sons with perinatal survival scores for sires

\begin{tabular}{lc}
\hline Number of sires & Number of sons \\
\hline 526 & 1 to 19 \\
25 & 20 to 39 \\
9 & 40 to 49 \\
3 & 50 to 59 \\
9 & 60 to 79 \\
5 & 80 to 99 \\
14 & 100 to 199 \\
4 & 200 to 299 \\
3 & 300 to 399 \\
1 & 400 to 427 \\
\hline
\end{tabular}

cows (Meyer et al., 2001a). These expected results were similar to the overall stillbirth results of $11 \%$ for primiparous cows and $5.7 \%$ for multiparous cows that were found for their data.

Perinatal survival is important because low rates of calf survival represent a financial and genetic loss to the dairy producer. This makes increasing perinatal survival a key issue for the growth and viability of a dairy. The objective of this study was to identify a bimodal pattern of inheritance for calf survival in progeny of Holstein bulls. This pattern of inheritance is indicative of an allele effect or a grandsire effect.

\section{MATERIALS AND METHODS}

Data on PTA for perinatal survival for the first parity of 8,678 sons of 599 sires were collected from 1984 through 1997 from the National Association of Animal Breeders (Columbia, MO) calving ease database, which included 7 Midwestern states (Iowa, Missouri, South Dakota, Nebraska, Oklahoma, North Dakota, and Kansas). We analyzed PTA estimated from first parity for bimodal distributions. Recently, Wiggans et al. (2008) validated the treatment of first and later parities as separate traits for analysis of stillbirths.

The distribution of sons per sire ranged from 1 sire with 427 sons to only 1 son each for 258 sires. Table 1 has the number of bulls with a particular number of sons for perinatal survival. From this data set, 39 bulls having at least 50 sons with first-parity records were selected for further analysis. Fifty sons per bull were chosen to allow close to 20 sons in each group for a bimodal bull. The PTA for perinatal survival was calculated using a sire-maternal grandsire (MGS) model. Reliability estimates for these values were not available because of the interactive nature of the analysis. The linear analysis for PTA was conducted at Iowa State University by C. L. Meyer and P. J. Berger (Meyer et al., 2001b).

Adding a factor of 0.89 to the PTA value and multiplying by 100 achieved a more biologically meaning-

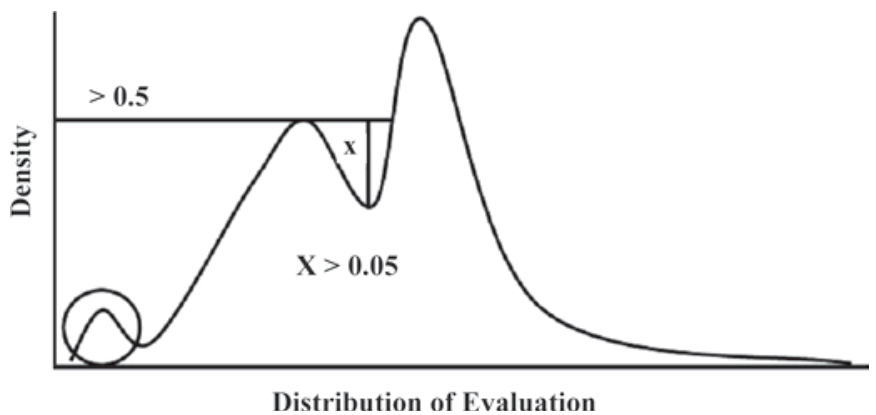

Figure 1. Visualization of criteria to be considered bimodal. Distribution was considered bimodal if 1) density of each peak was greater than 0.5 and 2) density of valley between peaks was at least 0.05 less than the shortest peak.

ful value for all of the sons. The factor of 0.89 was chosen because the value of 0.89 was the mean value of perinatal survival for the population as calculated by Meyer et al. (2001b). The mean value of 0.89 meant $89 \%$ survival or $11 \%$ mortality on average in primiparous cows. The result was multiplied by 100 to create the variable named percentage of PTA for perinatal survival (\% PTA PS). This biologically meaningful value was used in the kernel density estimation procedure in SAS (PROC KDE; SAS Institute, 2000) to separate the sons of sires with a bimodal distribution into 2 distinct groups. The oversmoothed option of the kernel density estimation procedure was a conservative method to define 2 or more significant peaks.

A set of criteria was established to determine if a distribution for a particular bull was bimodal. For a peak to be considered a peak, the density under the point of the peak must have been greater than 0.5. For a distribution to be considered bimodal, 2 peaks must have been present with a valley between the peaks. In addition, the density between the shorter peak and the valley needed to be greater than 0.05 . A visual representation is presented in Figure 1. A bimodal pattern after oversmoothing was considered significant. If a bull was found to have a bimodal distribution, the

Table 2. Example of creation of different combinations for a sire that has 5 sons

\begin{tabular}{lcc}
\hline & \multicolumn{2}{c}{ Number of sons } \\
\cline { 2 - 3 } Combination $^{1}$ & Low group & High group \\
\hline 1 & 0 & 5 \\
2 & 1 & 4 \\
3 & 2 & 3 \\
4 & 3 & 2 \\
5 & 4 & 1 \\
6 & 5 & 0 \\
\hline
\end{tabular}

${ }^{1}$ Only combinations 3 and 4 were valid in this example case because at least 2 sons are needed per bimodal group. 
Table 3. Number of bulls for distribution of sons for percentage of PTA for perinatal survival ${ }^{1}$

\begin{tabular}{|c|c|c|c|c|c|}
\hline \multirow[b]{2}{*}{ Number of bulls } & \multirow{2}{*}{$\begin{array}{l}\text { Range in number } \\
\text { of sons per bull }\end{array}$} & \multicolumn{3}{|c|}{ BWM } & \multirow[b]{2}{*}{ OS } \\
\hline & & 1 & 2 & 3 & \\
\hline 12 & 58 to 395 & $\mathrm{~N}$ & $\mathrm{~N}$ & $\mathrm{~N}$ & $\mathrm{~N}$ \\
\hline 8 & 55 to 226 & B & $\mathrm{N}$ & $\mathrm{N}$ & $\mathrm{N}$ \\
\hline 3 & 199 to 341 & B & B & $\mathrm{N}$ & $\mathrm{N}$ \\
\hline 5 & 61 to 141 & B & B & $\mathrm{N}$ & B \\
\hline 2 & 131 to 273 & $\mathrm{~T}$ & $\mathrm{~T}$ & B & B \\
\hline 9 & 50 to 427 & B & B & B & B \\
\hline
\end{tabular}

${ }^{1} \mathrm{BWM}=$ bandwidth multiplier; OS = oversmoothed method; $\mathrm{N}=$ normal; $\mathrm{B}=$ bimodal; $\mathrm{T}=$ trimodal. .

sons of the 2 groups of the bimodal distribution were analyzed separately. The 2 groups of sons found in a bimodal pattern were separated by calculating the $\mathrm{CV}$ within the groups. The procedure for separating out the groups was as follows:

1. The data were sorted in ascending order by PTA PS of the sons.

2. A program was created that distributed the sons into all possible combinations. The first combination had all sons in the low group and none in the high group (low group had a lower mean perinatal survival than the high group), the second combination had 1 son in the low group and the rest in the high group. An example of the different combinations can be seen in Table 2 . The number of possible combinations is equal to the number of sons plus one. Therefore, a bull with 5 sons had 6 possible combinations. However, only 2 of these 6 combinations could be possible bimodal combinations because at least 2 sons were needed in each of the bimodal groups.

3. Once all of the combinations were made, the CV was calculated for the 2 groups within each combination:

$$
C V_{i j}=\left[\frac{\sigma_{i j}}{\mu_{i j}}\right] \times 100,
$$

where $\sigma_{i j}=$ the standard deviation; $\mu_{i j}=$ the mean; $i=$ group $(1=$ low, $2=$ high $)$; and $j=$ combination.

4. The CV difference was calculated as the absolute value of the difference of the $\mathrm{CV}$ between groups within a combination $\left(\left|C V_{1 \mathrm{j}}-C V_{2 \mathrm{j}}\right|\right)$. The combination that gave the smallest $\mathrm{CV}$ difference was considered to be the combination that gave the proper distribution of the sons because this meant the variation relative to the mean between the 2 groups was most similar.
Once the sons were separated into low group and high group, the distributions of the MGS were analyzed. The hypothesis was tested to evaluate whether the grandsons of each MGS were distributed proportionally between the 2 groups.

\section{RESULTS AND DISCUSSION}

A bimodal distribution was found for 16 of the 39 bulls for perinatal survival using the oversmoothed
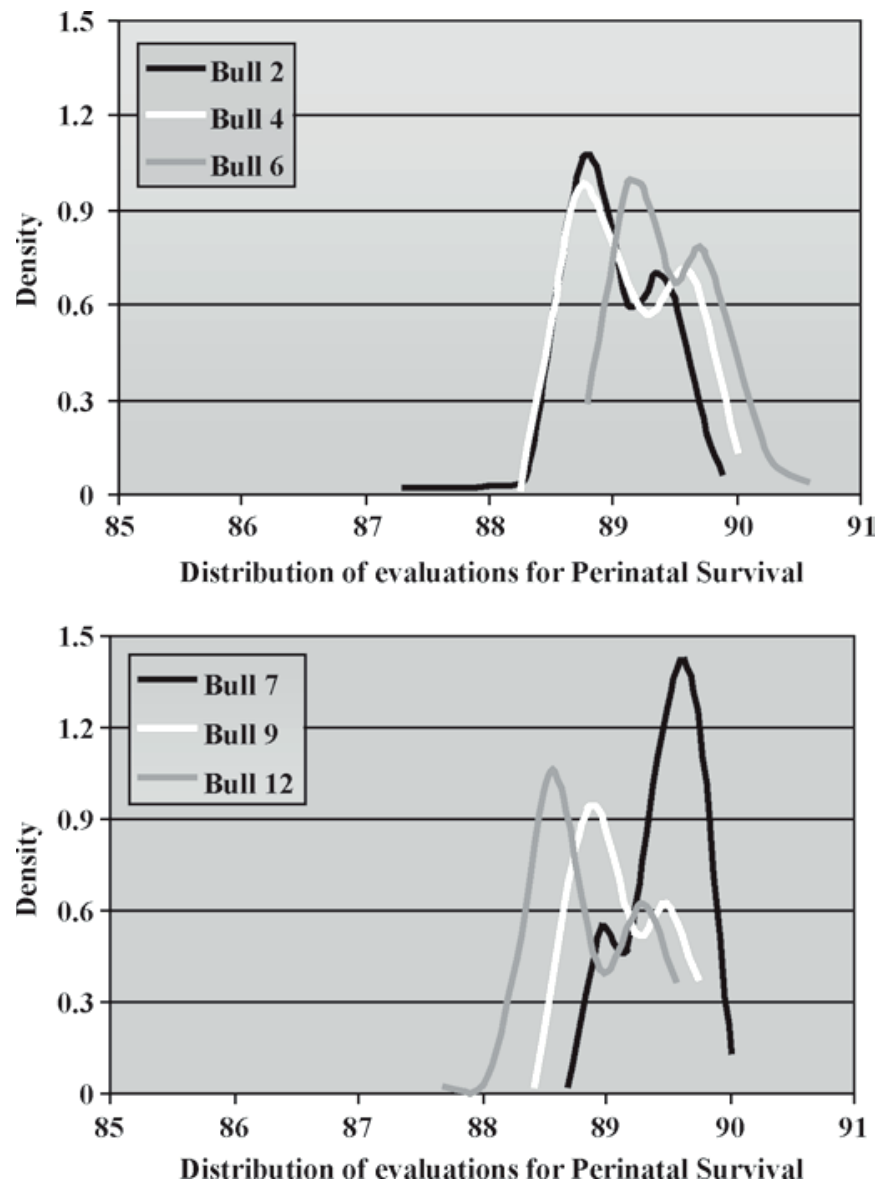

Figure 2. Oversmoothed graphs for bulls 2, 4, 6, 7, 9, and 12; these bulls have bull 28 as sire. 


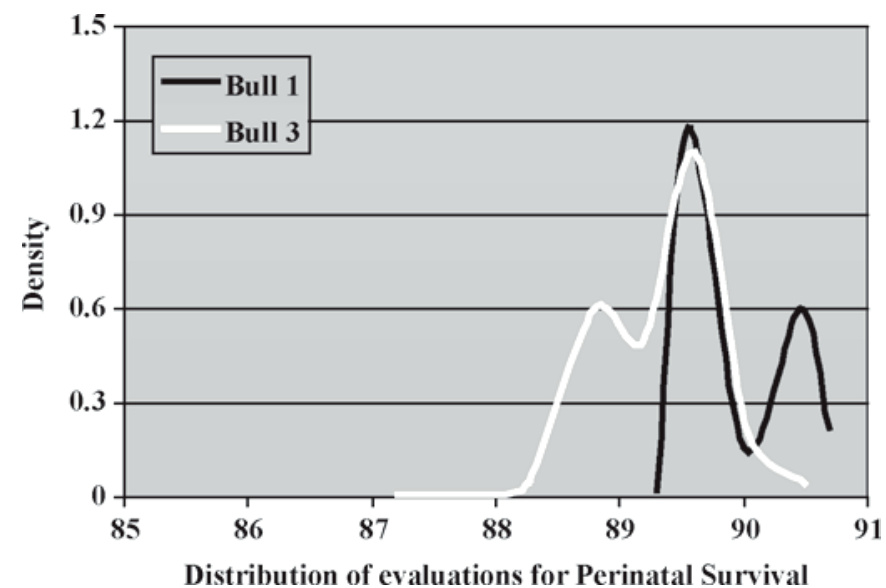

Figure 3. Oversmoothed graph for bulls 1 and 3; these bulls have bull 29 as sire.

method. Distributions for sons of the remaining 23 bulls were normal. Table 3 has a summary of the distributions of sons of 39 bulls. This table shows the identity, whether normal, bimodal, or trimodal, of the density graphs for each bull for each bandwidth multipliers and for the oversmoothed method. Figure 2 shows the oversmoothed graphs for bulls 2, 4, 6, 7, 9, and 12. These bulls are grouped together because they all have bull 28 as a sire. These bulls also have similar distributions for perinatal survival. They all have 1 peak around 89 . Figure 3 shows the oversmoothed graph for bulls 1 and 3 . These bulls are grouped together because they have bull 29 as a sire. Like the bulls in Figure 2, these bulls also have a similar distribution for perinatal survival. They both have a peak around 89.5. Figure 4 shows the oversmoothed graph for bulls 5 and 13. These bulls

Table 4. Means of sons and percentage of PTA for perinatal survival for each of the 16 bimodal bulls

\begin{tabular}{lccc}
\hline & \multicolumn{2}{c}{ Total } & \\
\cline { 2 - 3 } Bull & Sons, n & Mean & Bull PTA, \% \\
\hline 1 & 273 & 90.0 & 91.1 \\
2 & 79 & 89.0 & 89.4 \\
3 & 192 & 89.5 & 89.7 \\
4 & 117 & 89.2 & 89.5 \\
5 & 75 & 89.8 & 90.7 \\
6 & 63 & 89.5 & 90.3 \\
7 & 116 & 89.5 & 89.8 \\
8 & 70 & 88.8 & 88.6 \\
9 & 61 & 89.2 & 89.6 \\
10 & 72 & 88.6 & 88.0 \\
11 & 95 & 89.3 & 89.7 \\
12 & 116 & 88.9 & 88.9 \\
13 & 131 & 89.7 & 90.5 \\
14 & 141 & 90.4 & 91.4 \\
15 & 427 & 87.8 & 86.6 \\
16 & 50 & 88.4 & 87.6 \\
\hline
\end{tabular}

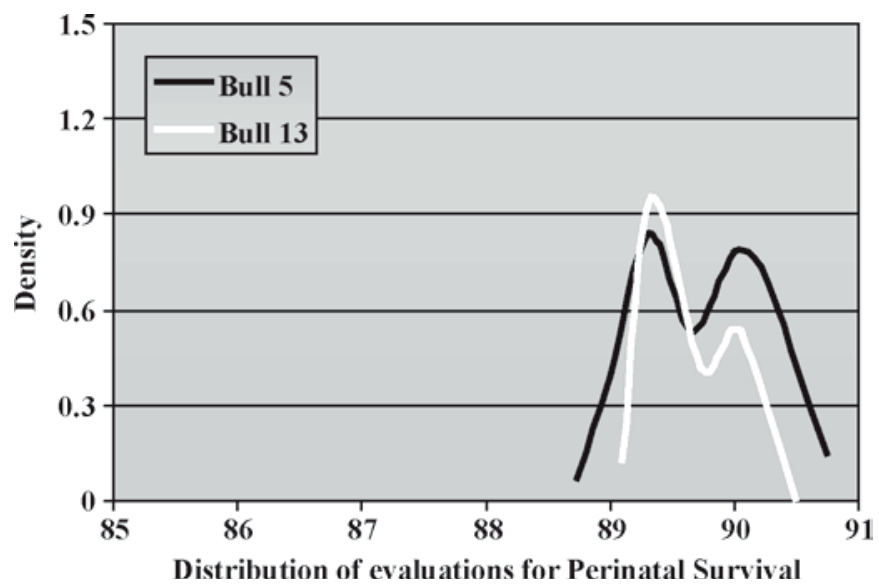

Figure 4. Oversmoothed graph for bulls 5 and 13; these bulls have bull 26 as sire.

are grouped together because they have bull 26 as a sire. They also have similar distributions of perinatal survival and have a peak around 90 . Figure 5 shows the oversmoothed graph for bulls 15 and 16 . These 2 bulls are maternal brothers. Distribution of sons of bull 15 covers a wide range of survival. Bull 15 has 13 MGS with greater than 5 grandsons; this indicates that he was mated to a diverse group of dams, which could account for the large spread in PTA PS for his sons (Figure 5). Graphs of bulls 8, 10, 11, and 14 are not shown because these bulls are not related at the parent level; however, bull 19, bull 21, or both appear somewhere in all of their pedigrees. Table 4 has the mean \% PTA PS of sons and \% PTA PS for each of the 16 bimodal bulls. The correlation between the PTA PS of the bull and the mean PTA PS of his sons in Table 4 is 0.98. A correlation of 1 is expected because the PTA PS of the

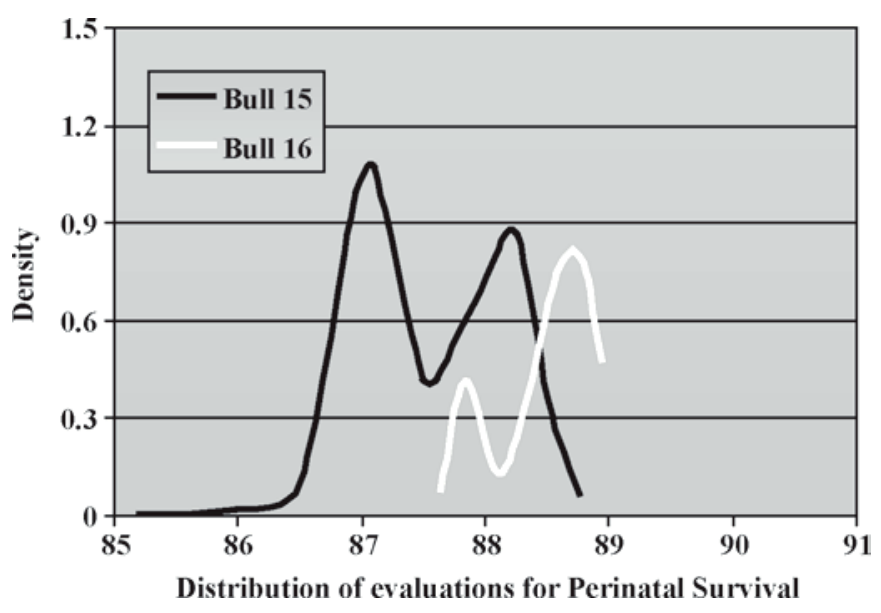

Figure 5. Oversmoothed graph for bulls 15 and 16; these bulls have the same dam. 
Table 5. Low and high groups for the bimodal bulls with sons having PTA for perinatal survival

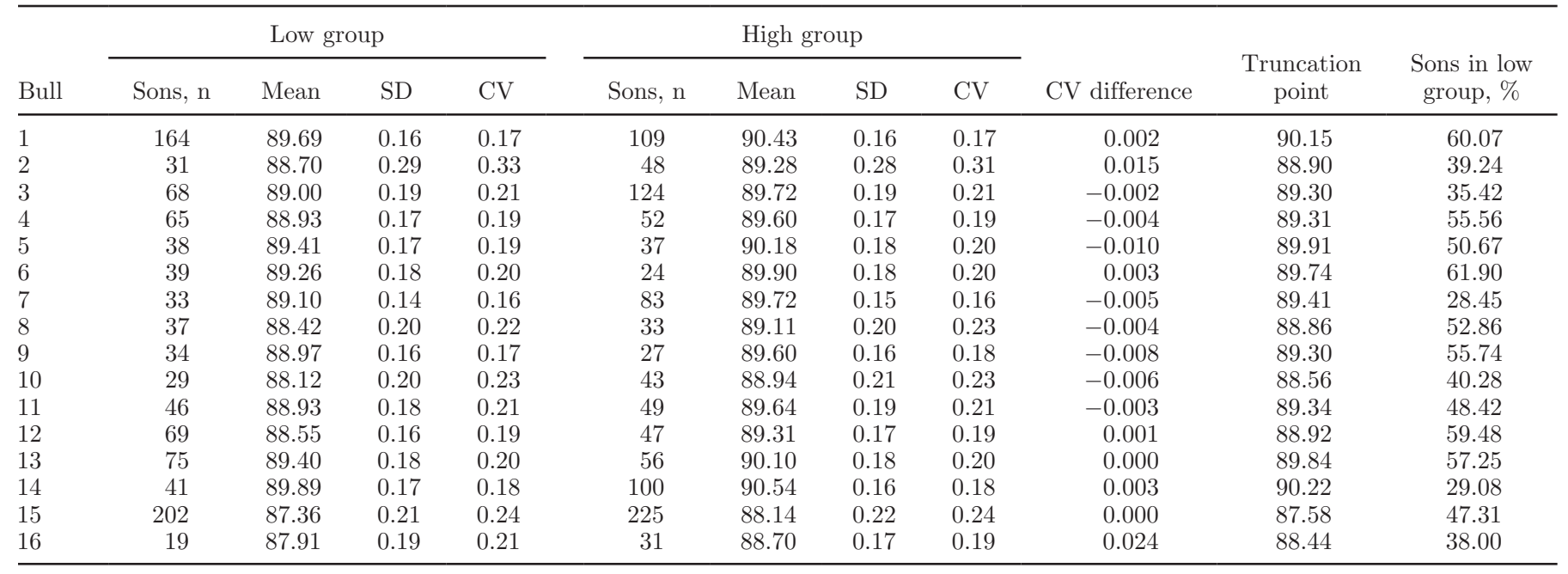

bull should include the information from his sons. For each of the 16 sires having a bimodal distribution, 2 groups of sons were separated using the $\mathrm{CV}$ technique described in the Materials and Methods. The number of sons for each of the bimodal bulls and the number of sons that fall into each of the 2 groups is summarized in Table 5.

The MGS, having greater than 5 grandsons, were ranked based on their PTA PS. These rankings were used to numerically identify the MGS from 1 (best survival) to 41 (worst survival). Four MGS had only descendants with bimodal bulls, 13 MGS had descendants with both normal and bimodal bulls, and 24 MGS had descendants with only normal bulls.

Table 6 indicates whether grandsons of a particular MGS were in low, high, or both groups. A minimum of 5 grandsons was required for MGS to be counted. The grandsons of the MGS appeared to be localized to one group or the other. The group containing the majority of the grandsons corresponded to the putative location of the particular MGS with respect to PTA PS of MGS (Schlesser, 2004). For example, the MGS with most grandsons in the low group had low PTA PS.

The distribution of the MGS was also analyzed for the 23 normally distributed bulls. This was done to verify that the MGS were not localized to different halves of the normal distribution like they were for the bimodal bulls. The distribution of MGS for bimodal and normal bulls is summarized in Table 7. The distribution of the MGS for the bimodal bulls had more sons in either the low or the high group, whereas the distribution of the normal bulls had more sons in both groups. The $\chi^{2}$ statistic was calculated to determine if the distribution seen in the bimodal bulls was significantly different from the distribution seen in the normal bulls. The $\chi^{2}$ statistic of 23.3 indicates that the distribution of the MGS was significantly different $(P<0.01)$ between bimodal and normal bulls.

Figure 6 was created to determine the distribution of the sons for each of the 39 bulls having perinatal survival data and the 41 MGS. From this figure, it appears that the MGS with daughters mated to bimodal bulls (first 16) either have high survival or low survival. For example, MGS 2, 3, 4, 6, and 7 appear to have low survival and MGS 35, 38, 39, 40, and 41 have high survival. It also appears that the MGS for the normally distributed bulls are more equally distributed. Figure 7 was created to check the relationship of age between the sires and MGS. As expected, this graph is almost linear, indicating that sons from younger sires

Table 6. Distribution of maternal grandsires (MGS) for the bimodal bulls for perinatal survival

\begin{tabular}{|c|c|c|c|c|c|c|c|c|c|c|c|c|c|c|c|c|}
\hline Number of MGS & \multicolumn{16}{|c|}{ Bull } \\
\hline With 5 or more descendants & 9 & 5 & 6 & 6 & 4 & 5 & 7 & 5 & 4 & 5 & 5 & 6 & 8 & 9 & 13 & 3 \\
\hline With grandsons in both groups & 1 & 2 & 3 & 1 & 0 & 1 & 2 & 1 & 0 & 1 & 0 & 1 & 1 & 4 & 6 & 0 \\
\hline With grandsons in high group only & 3 & 1 & 1 & 2 & 2 & 1 & 3 & 1 & 2 & 2 & 2 & 2 & 3 & 4 & 6 & 2 \\
\hline
\end{tabular}


Table 7. Summary of distribution of maternal grandsires (MGS) for bimodal and normal bulls

\begin{tabular}{lccc}
\hline Item & $\begin{array}{c}\text { MGS, n, with grandsons } \\
\text { in low group only }\end{array}$ & $\begin{array}{c}\text { MGS, n, with grandsons } \\
\text { in both groups }\end{array}$ & $\begin{array}{c}\text { MGS, n, with grandsons } \\
\text { in high group only }\end{array}$ \\
\hline Bimodal & 39 & 24 & 37 \\
Normal & 38 & 94 & 32 \\
\hline
\end{tabular}

have younger MGS and sons from older sires have older MGS. The bimodal bulls fall on the right side of this graph, indicating that they are young bulls. The fact that all of the bimodal bulls are young indicates that the bimodal distribution may be a consequence of the model; however, it is hard to definitively determine the cause of this without reanalyzing the data with a different model. Also, because the bulls are younger, the bimodal distribution may arise from a limited number of sons. However, it was found that the bimodal bulls tended to have similar numbers of sons as the older bulls.
Both within- and across-family studies have the potential to further genetic advancement. Current methods of genomic selection make successful predictions based on across-family similarities. Detection of bimodal distributions within some families identifies that not all families are the same. Also, the distribution of sire-MGS combinations illustrated in Figures 6 and 7 indicates that matings may not be occurring at random. Across-family approaches to genomic selection assume random mating. Impact of the validity of assumptions of across-family studies is warranted. Within-family studies may provide insights to validity.

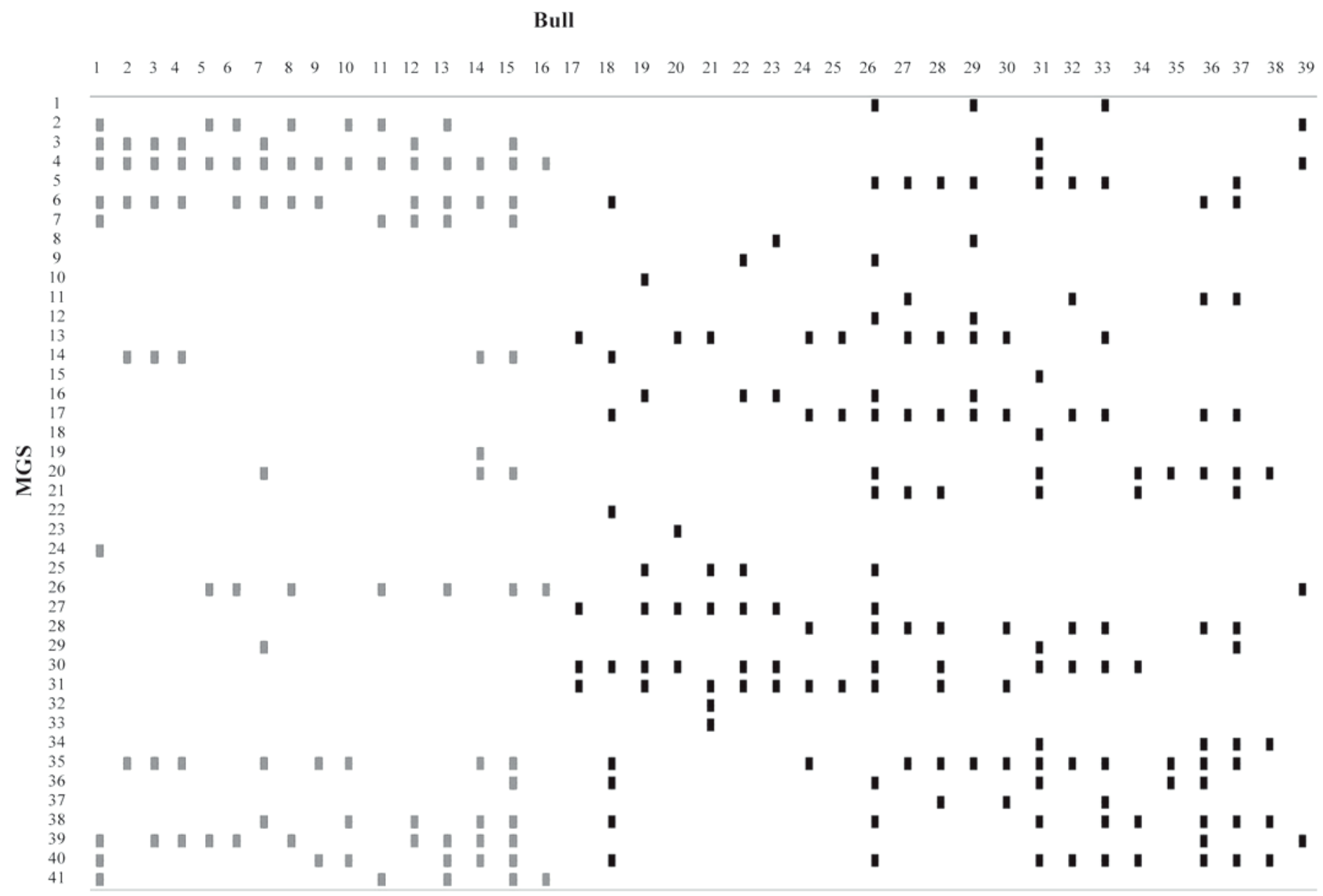

Figure 6. Distribution of sons between the 39 bulls having PTA for perinatal survival and the 41 maternal grandsires (MGS). The first 16 bulls are bimodal. Maternal grandsires are listed in order of PTA for perinatal survival from highest (top) to lowest (bottom) survival. 


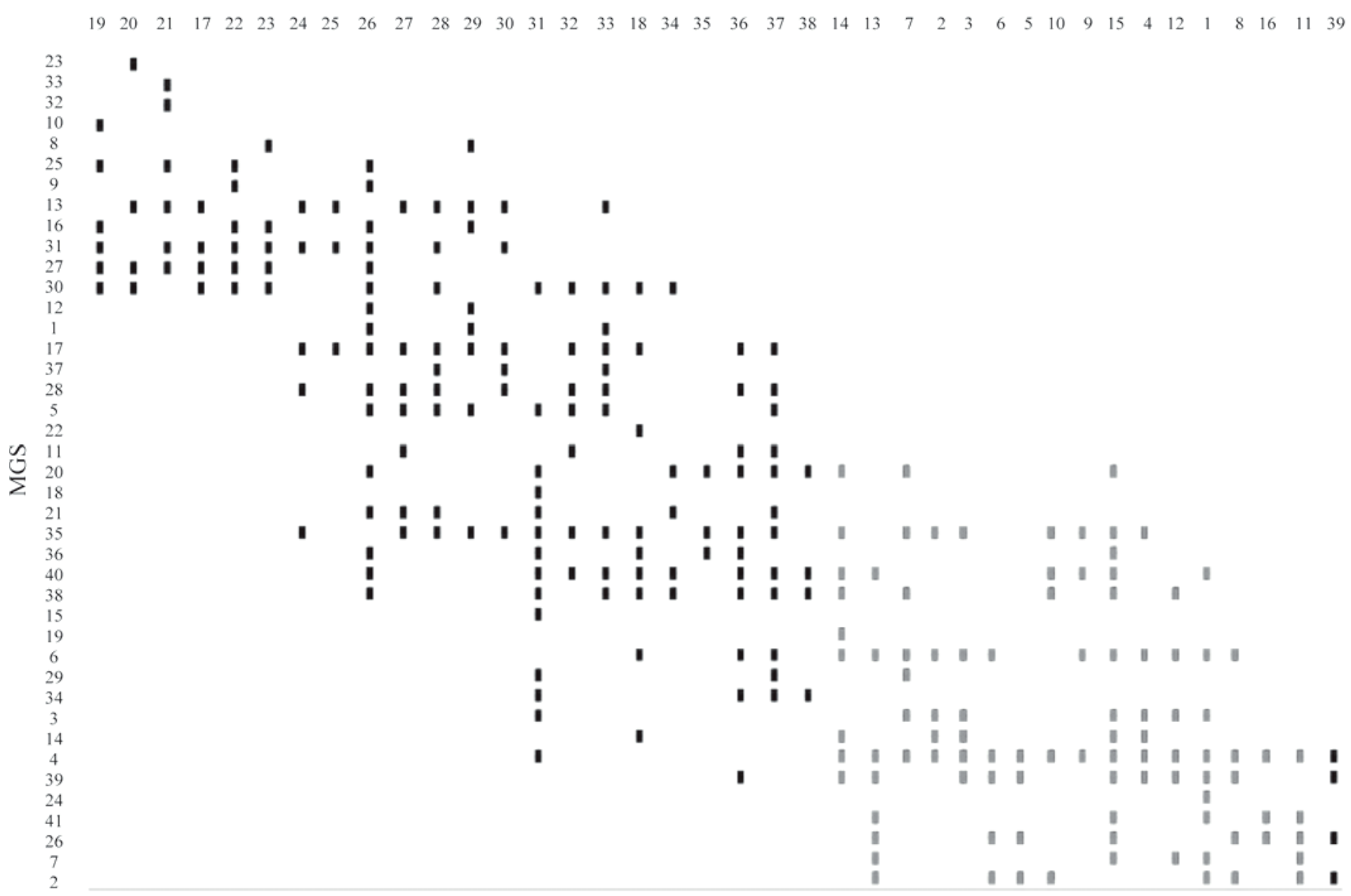

Figure 7. Ordered distribution of sons among 39 bulls having PTA for perinatal survival and the 41 maternal grandsires (MGS). Bulls are ordered from oldest (left) to youngest (right). Maternal grandsires are ordered from oldest (top) to youngest (bottom).

Besides being able to calculate the average allele effect from this data, it was also possible to estimate the gene frequencies in the population. To do this, the 16 bulls that had a bimodal distribution were considered to be heterozygous (Aa). The 23 normally distributed bulls were considered to be homozygous (AA, aa). The normally distributed bulls that had mean perinatal survival less than the average perinatal survival of the bimodally distributed bulls were considered to be homozygous-recessive, and those having perinatal survival scores above the average of the bimodally distributed bulls were considered to be homozygous-dominant. The mean \% PTA PS for the 16 heterozygous bulls was 89.22. Seven normally distributed bulls had perinatal survival scores less than 89.22, and 16 had PTA PS greater than 89.22. With this information, the gene frequency of A (allele for greater survival) was calculated to be 0.62 , and the gene frequency of a (allele for less survival) was calculated to be 0.38 .

\section{CONCLUSIONS}

The objective of this research was to better understand the genetic influence of perinatal survival. The method to accomplish this objective was to characterize the distribution, unimodal, or bimodal for particular sires.

Kernel density estimation graphs, created using PROC KDE in SAS, gave visual representations of the distribution for sons of bulls. Sixteen of the 39 bulls analyzed for perinatal survival had a bimodal distribution. The 16 bulls found to have a bimodal distribution for PTA PS were young bulls. The fact that all of the bimodal bulls were young may indicate a systematic influence or a disturbing change of reduced allele frequency of favorable alleles over time.

The characteristics of the distribution of grandsons between the normally distributed and bimodally distributed bulls having PTA PS were not consistent. The 
distribution of MGS for the bimodal bulls was nonrandom, and the distribution of MGS for the normal bulls appeared random. The nonrandom pattern of performance from the MGS and descendants may indicate that the bimodal pattern of inheritance is caused by MGS and not by an allele effect from the sire. However, the allele effect from the sire for perinatal survival was estimated at 0.35 . The allele frequency for perinatal survival was 0.62 for allele $\mathrm{A}$, increased perinatal survival, and 0.38 for allele a, decreased perinatal survival.

\section{REFERENCES}

Cole, J. B., G. R. Wiggans, and P. M. VanRaden. 2007a. Genetic evaluation of stillbirth in United States Holsteins using a sirematernal grandsire threshold model. J. Dairy Sci. 20:2480-2488.

Cole, J. B., G. R. Wiggans, P. M. VanRaden, and R. H. Miller. 2007b.

Stillbirth (co)variance components for a sire-maternal grandsire threshold model and development of a calving ability index for sire selection. J. Dairy Sci. 90:2489-2496.
Meyer, C. L., P. J. Berger, and K. J. Koehler. 2000. Interactions among factors affecting stillbirths in Holstein cattle in the United States. J. Dairy Sci. 83:2657-2663.

Meyer, C. L., P. J. Berger, K. J. Koehler, J. R. Thompson, and C. G. Sattler. 2001a. Phenotypic trends in incidence of stillbirth for Holsteins in the United States. J. Dairy Sci. 84:515-523.

Meyer, C. L., P. J. Berger, J. R. Thompson, and C. G. Sattler. $2001 b$. Genetic evaluation of Holstein sires and maternal grandsires in the United States for perinatal survival. J. Dairy Sci. 84:1246-1254.

Patterson, D. J., R. A. Bellow, P. J. Burfening, and J. B. Carr. 1987. Occurrence of neonatal and postnatal mortality in range beef cattle. I. Calf loss incidence from birth to weaning, backward and breech presentations and effects of calf loss on subsequent pregnancy rate of dams. Theriogenology 28:557-571.

SAS Institute. 2000. SAS Software Release, Version 8.1. SAS Institute Inc., Cary, NC.

Schlesser, H. N. 2004. Graphical approach to evaluate genetic estimates of fertility and survival. MS Thesis. University of Illinois, Urbana.

Thompson, J. R., A. E. Freeman, P. J. Berger, and M. L. Martinez. 1981. A survey of dystocia and calf mortality in five dairy breeds. J. Dairy Sci. 64(Suppl. 1):81. (Abstr.)

Wiggans, G. R., J. B. Cole, and L. L. M. Thornton. 2008. Multiparity evaluation of calving ease and stillbirth with separate genetic effects by parity. J. Dairy Sci. 91:3173-3178. 